\title{
Research on the Construction and Application of Chinese-English Parallel Corpus of Red Tourism: Taking Hebei Province as a Case
}

\author{
Caiqiao Huo $^{1,2}$, Guangtian Zhang² \\ ${ }^{1}$ School of Chinese Language and Literature, Hebei University, Baoding, China \\ ${ }^{2}$ College of Foreign Language Education and International Business, Baoding University, Baoding, China \\ Email: goofyhuo@163.com
}

How to cite this paper: Huo, C. Q., \& Zhang, G. T. (2022). Research on the Construction and Application of Chinese-English Parallel Corpus of Red Tourism: Taking Hebei Province as a Case. Creative Education, 13, 557-564. https://doi.org/10.4236/ce.2022.132033

Received: January 20, 2022

Accepted: February 15, 2022

Published: February 18, 2022

Copyright ( 2022 by author(s) and Scientific Research Publishing Inc. This work is licensed under the Creative Commons Attribution International License (CC BY 4.0).

http://creativecommons.org/licenses/by/4.0/ (c) (i) Open Access

\begin{abstract}
Red tourism is the activities which enable tourists to realize the importance of the revolutionary spirit and receive revolutionary traditional education by visiting revolutionary memorials. Red tourism attracts more and more tourists with its unique cultural connotation. Its English translation is the key to opening up overseas markets. Building a Chinese-English bilingual corpus of red tourism is of great benefit to English translation research. This article first summarizes the theoretical and practical significance of the construction of a Chinese-English bilingual corpus of red tourism; then discusses the urgency of constructing a bilingual corpus of red tourism from two perspectives: improving the quality of red tourism English translation and telling red stories of Hebei; on this basis, the steps of corpus construction are proposed; finally, the application of the corpus is explained from three levels: serving for the study of the theory and practice of Chinese-English translation, serving for the teaching of Chinese-English translation, and serving for the language and cultural comparative research.
\end{abstract}

\section{Keywords}

Red Tourism, Bilingual Corpus, Construction, Application

\section{Introduction}

Red tourism refers to a kind of activity which organizes tourists to visit revolutionary memorial sites and monuments so as to make tourists realize the significance of the revolutionary spirit and receive the traditional education of the revolution. The red culture of the Communist Party of China has a long tradi- 
tion, and red tourist attractions carry a rich red culture. With the vigorous promotion of red tourism by the Central Committee of the Communist Party of China, there has been a continuous upsurge of red tourism across the country. Provinces and cities are scrambling to develop and plan red tourism, which has also aroused the interest of a large number of investors and has stimulated the development of red tourism (Wei, 2018). This study investigated the status quo of the translation of red tourism resources in Hebei Province and found that there are mistranslations, omissions, grammatical errors, multiple translations, lack of cultural information, and even spelling errors in some translated texts of red tourism resources. The red tourism resources in Hebei Province are supreme, iconic and unique in the whole country. The problems existing in the translation of these red tourism resources have seriously affected the image and level of these red scenic spots. Therefore, it is necessary to build a Chinese-English parallel corpus of red tourism resources to standardize the translation of red tourism resources in Hebei. This study takes Hebei Province as an example to discuss the construction method and application prospect of a bilingual corpus of red tourism resources.

\section{Research Background}

Corpus first originated in the United States in the 1960s, symbolized by the completion and application of the Brown corpus. Sinclair gives a representative definition of corpus earlier: A corpus is a collection of naturally occurring language texts that describe the states and variants of a language (Sinclair, 1991). Hunston defines a corpus more specifically as a collection of naturally occurring languages collected for linguistic research, which can be a few sentences, or a collection of texts or recordings (Hunston, 2002). Corpus linguistics is a new discipline that studies language phenomena and laws based on a large number of objective corpora collected (Yang, 2002). The corpus method was introduced into China in the early 1990s, and since then domestic scholars have begun to study corpus (Chang \& Yu, 2009). Corpus makes translation research more objective and sufficient by virtue of real and reliable data and empirical research methods. Baker was the first to apply corpus to translation research. He put forward the concept of corpus translation studies, and expounded its development prospects (Baker, 1993).

A bilingual parallel corpus is a kind of corpus, which consists of the original language of a language and its target language with parallel correspondences at the word, sentence, and paragraph levels. A bilingual parallel corpus contains a large number of bilingual examples, which are widely used in machine translation, translation teaching, text translation, dictionary compilation and other fields (Wang, 2004). Establishing parallel corpora and conducting research is one of the important representations, which represents the current development trend of translation research, language contrast research and foreign language teaching research, namely the corpus-based method (Zhen \& Zhang, 2004). A parallel corpus is to input the completely corresponding texts of the two lan- 
guages in the computer, and then compare and analyze how the two languages express the same contents (Xiao \& Dai, 2011).

As scholars engaged in translation research and language contrast research gradually realized the importance of parallel corpora, the earliest domestic discussions on tourism corpus were Li Dechao, Wang Kefei, etc. Their papers expounded the design concept of English-Chinese tourism corpus, and discussed the research and teaching of tourism translation (Li \& Wang, 2010). Their research has important guiding significance for the construction of tourism corpus. But its research is only limited to a certain historical period and lacks continuous and dynamic research. In addition, the research application is only limited to the tourism translation in Hong Kong, and it is rarely involved in many domestic tourism materials. Xiao \& Chen (2012) built Hengyang Chinese-English Parallel Tourism Corpus, which greatly promoted the development of the local tourism industry and cross-cultural communication. In recent years, many domestic researchers have combined the construction of parallel corpus with local tourism. It advocates to excavate and organize the local red cultural heritage, and build an English-Chinese bilingual corpus, so that the local red cultural heritage can be inherited (Wei, 2018; Wu, 2020). In view of this, it is of great significance to construct the English-Chinese parallel corpus of Hebei red tourism and conduct related research.

\section{The Significance of the Construction of Hebei Red Tourism Corpus}

Hebei has a special geographical location, a long history and profound cultural heritage. The red revolutionary culture formed during the revolutionary war is distributed throughout the urban and rural areas of Hebei. These cultures have distinct themes and their own characteristics (Tian \& Li, 2021). Its significance mainly lies in:

\subsection{Theoretical Significance}

First of all, the corpus to be constructed is a multimodal parallel corpus that integrates various information such as audio, video and text corpus, which will provide a good paradigm and convenient entry for the dissemination and research of Hebei red spirit. Secondly, the completed corpus provides a large number of well-documented real corpora. In addition to red tourism resources and red education bases, it also includes local dialects and customs, local red culture and history, and materials of red art works. It will also serve translation research, red cultural studies, local studies, etc. In particular, translation studies are paying more and more attention to localization characteristics. Translation studies and cultural studies based on corpus comparisons are also the frontiers of international related academic fields. The proposed corpus can provide a more comprehensive and authoritative resource base for the translation theory and practice research and cultural research of various research institutions in the 
province and even the whole country.

\subsection{Practical Significance}

Hebei Red Resource Corpus has positive significance for Hebei's external publicity, internationalization, tourism brand promotion, and retention of revolutionary historical materials. As an old revolutionary base, governments at all levels have been paying attention to the development of Hebei. The central government also proposed to carry forward the spirit of revolution and give full play to the advantages of red resources. Establishing a corresponding red resource corpus and seizing the opportunities of the times are also in response to the overall national development plan and the needs of the development of the times. The proposed corpus standardizes and unifies the translation of related texts and terms, and integrates and categorizes the red resource culture in Hebei, which covers many red resources that have not been fully explored and publicized. It has good practical significance for promoting urban branding and preserving the red resources of diversity. As far as translation teaching and localization translation services of Hebei tourism are concerned, the authority and authenticity of the corpus are irreplaceable by general auxiliary tools and manpower. This corpus can accommodate a large number of authoritative Chinese and English examples, which can be applied to translation teaching and promote the cultivation of high-quality translators. In addition, the bilingual corpus provides a good paradigm and reference for the future red culture translation project.

\section{The Urgency of Red Tourism Corpus Construction}

\subsection{Improvement of the Quality of English Translation of Relevant Texts of Hebei Red Tourism and Red Education Base}

Hebei Province is full of red tourist attractions and red education bases, such as Xibaipo Red Tourist Area, North China Military Region Martyrs Cemetery, the former site of the Eighth Route Army $129^{\text {th }}$ Division Headquarters, Langya Mountain, Jinji Luyu Martyrs Cemetery, Ranzhuang Tunnel Battle Site, Jin Chaji Border Area Revolutionary Memorial Hall and other scenic spots or old sites; as well as hero-themed education bases such as Li Dazhao Memorial Hall, Rong Gaotang Memorial Hall, Dong Cunrui Martyr Memorial Hall, Ma Benzhai Memorial Hall, Guo Shoujing Memorial Hall, Li Shaochun Memorial Hall and so on. However, through on-the-spot investigation of some red tourist attractions and educational bases, it is found that the translation problems of relevant texts are prominent, such as cultural mistranslation, omission of translation, grammatical errors, pragmatic errors, and inconsistencies in the translation of proper names. In the process of creating the corpus, the researchers will sort out these corpora, and carry out repeated proofreading and revision to ensure the standardization and accuracy of the corpus. The proposed corpus will also supplement the missing translations, which will improve the English translation of red resources in Hebei to a certain extent. 


\subsection{Importance to Tell Red Stories in English}

The stories of Red Revolution in Hebei are rich in content. The main purpose is to tell the stories of "people, objects, events, and souls" well, and use the stories to encourage people in contemporary society. "People" refers to the people with lofty ideals and revolutionary martyrs with revolutionary spirits, including not only heroes who shine in the annals of history, but also ordinary revolutionary masses. For example, the five heroes of Langya Mountain, the revolutionary martyr Dong Cunrui, the anti-Japanese hero Haiwa, the cow herding baby Wang Erxiao, and the soldier Zhang Ga, etc. "Objects" refer to the relics and memorials of the Revolutionary War, the former residences of historical figures, and the objects used by revolutionary soldiers during their lifetimes. For example, the Li Dazhao Memorial Hall, the former site of the $129^{\text {th }}$ Division Headquarters of the Eighth Route Army in Shexian County, the Five Heroes Exhibition Hall in Langya Mountain, the Ranzhuang Tunnel War Memorial Hall, the Bethune Ketihua Memorial Hall, the Exhibition Hall of the Chinese People's Anti-Japanese Military and Political University (hereinafter referred to as the Anti-Japanese University Exhibition Hall), Chengnanzhuang Jinchaji Border Revolution Memorial Hall, Xibaipo Revolutionary Memorial Hall, Shanxi-Hebei-Luyu Revolutionary Memorial Hall, etc. "Events" refer to historical events that have a major impact on history or influential revolutionary heroic deeds. For example, the student movement of the Second Division of the Red Division in Baoding, and the people of the Jizhong Plain tunnel warfare. "Soul" refers to the spirit and integrity formed in the revolutionary struggle. For example, Xibaipo spirit, Bethune spirit, etc. It is necessary to dig deep into the historical stories of the revolutionary ancestors' unremitting struggle for revolutionary ideals, and grasp the ideals and beliefs that run through the entire revolutionary process. Through the storytelling of "people, objects, events, and souls", people can deeply understand that a happy life is hard-won, which can strengthen the ideals and beliefs of socialism, and improve the centripetal force and cohesion of the nation. To tell the story of the red revolution well, it is necessary to highlight the lofty spirit of hard work, selfless dedication and fearlessness contained in the story; it is necessary to clearly explain the ideological connotation, historical value and implication of the times of the revolutionary spirit, so as to encourage people to always maintain a sense of urgency and to cultivate the feelings to family and country. Through each red story, the history of the struggle of the party and the history of the founding of the People's Republic of China is clearly displayed, so that the people can truly appreciate the original intention and mission of the Communist Party of China (Tian \& Li, 2021).

\section{Construction of Chinese-English Parallel Corpus of Hebei Red Resources}

The proposed corpus is a Chinese-English parallel corpus that can be used for Hebei revolutionary spirit research and red culture publicity. The database is 
composed of several sub-databases, including the original corpus of about 1 million words and a corpus of 3 million words (a collection of representative corpora of red tourist attractions, red education bases, and red cultural heritage).

In the process of building the database, the relevant theories of corpus construction and research at home and abroad can be used, and effective representative samples and research tools can be selected to carry out research work. In the process of project implementation, technical tool design and platform construction are carried out. The tools mainly include database building tools and retrieval tools, while the platforms include corpus offline retrieval platforms and network retrieval platforms.

The construction process of Chinese-English Parallel Corpus of Hebei Red Resources can be designed as follows: 1) The principles of corpus construction; 2) The selection and sampling of the corpus; 3 ) The proofreading and entry of the corpus; 4) The word segmentation and labeling of the corpus; 5) Alignment of corpus; 6) Generation of online corpus retrieval platform and terminology database.

The following three channels can be considered for the language material selection of this corpus: firstly, collecting corpus from authoritative official publications (books, periodicals, audio and video, etc.); secondly, selecting from non-publications (e.g. websites, tourism promotion materials), in which the accuracy of the materials needs to be paid attention to and authoritative proofreading and revision should be conducted; thirdly, for some language materials with incomplete translations, more mistranslations or no corresponding English, the researcher can choose relative materials and produce the electronic text by themselves. In order to ensure the representativeness and authority of sampling, stratified sampling is used for sampling, and after three different types of texts are identified in the Chinese corpus, a random sampling method is used to obtain samples in each category of texts. Only lexical annotation of the corpus is performed in the study, not syntactic annotation and semantic annotation.

\section{Application of the Chinese-English Parallel Corpus of Red Tourism}

On the one hand, translation research pays more and more attention to the characteristics of localization. The construction of the bilingual corpus of red tourism in Hebei Province is a pioneering attempt to promote translation localization research. It contains detailed bilingual materials, diversifies the translation corpus, and provides a wealth of theoretical and practical examples for foreign publicity research. On the other hand, the examples in this corpus are all objective and well-documented real corpora, based on which new technical software can be developed, ways to expand the scale of the corpus can be explored, and in-depth translation of red tourism-type texts can be carried out. Technicians can create practical tools, use information means to promote the red tourism bilingual corpus, and provide a network search portal, that is, using the red tour- 
ism corpus as the material basis and the online search engine as an intermediary. The corpus in the form of a local area network can be converted into online information search forum, through which multiple corpora that are not closely related to each other are properly linked. In this way, bilingual materials and comprehensive coverage of online information on red tourism can be shared to build an online corpus with rich materials at last.

\section{Conclusion}

The Hebei Chinese-English bilingual corpus of Red Tourism built through corpus extraction, corpus sampling, corpus coding and corpus processing alignment will set a precedent for the construction of a corpus of red tourism in the province, and is conducive to promoting translation talents with localization and globalization characteristics, so as to promote the development of the local red tourism industry and the external publicity of red culture. At the same time, it is of great theoretical and practical value to apply the corpus to translation, teaching and comparative study of language and culture. In addition, with the vigorous development of the corpus, combined with advanced information technology, scholars can extend the corpus to other specific fields to provide more objective data for the development of corpus linguistics.

\section{Acknowledgements}

This paper is under the project of Research on Hebei Tourism Document Translation Based on English-Chinese Diachronic Parallel Corpus (project No. 20210201244) which is sponsored by Social Science Association of Hebei Province and the project of Research on the Application of Corpus in English Writing Feedback (project No. 2020YYJG051) which is sponsored by Hebei Provincial Department of Education. All the project members made their contributions to the paper. I also thank all my friends and classmates who work in different universities, which made the investigation possible.

\section{Conflicts of Interest}

The authors declare no conflicts of interest regarding the publication of this paper.

\section{References}

Baker, M. (1993). Corpus: Linguistics and Translation Studies: Implications and Applications. In M. Baker, G. Francis, \& E. Tognini-Bonelli (Eds.), Text and Technology: In honor of John Sinclair. Amsterdam (pp. 233-250). John Benjamins. https://doi.org/10.1075/z.64.15bak

Chang, B. B., \& Yu, S. W. (2009). Corpus Technology and Its Application. Foreign Languages Research, 5, 43-51.

Hunston, S. (2002). Corpora in Applied Linguistics. Cambridge: Cambridge University Press. https://doi.org/10.1017/CBO9781139524773

Li, D. C., \& Wang, K. F. (2010). Development and Application of Bilingual Corpora of 
Tourism Texts: A New Approach. Modern Foreign Languages, 1, 46-54, 109.

Sinclair, J. (1991). Corpus, Concordance, Collocation. Oxford: Oxford University Press.

Tian, H. J., \& Li, H. J. (2021). Reearch on the Construction of Hebei Red Evolutionary Culture Communication System. Journal of Hebei University (Philosophy and Social Science), 4, 55-63.

Wang, K. F. (2004). Bilingual Correspondence Corpus: Development and Application. Beijing: Foreign Language Teaching and Research Press.

Wei, L. (2018). Construction and Application of Red Tourism Bilingual Corpus-Taking Anhui Province as an Example. Journal of Hubei University of Economics (Humanities and Social Sciences), 4, 124-126.

$\mathrm{Wu}$, L. H. (2020). Concept for the Construction of the Dabie Mountain Red Resources Chinese-English Parallel Corpus. Journal of Ningbo Polytechnic, 8, 93-97.

Xiao, G. S., \& Chen, X. (2012). Construction and Application of Chinese-English Parallel Corpus for Tourism. Journal of Hunan Institute of Science and Technology, 10, 163-165.

Xiao, Z. H., \& Dai, G. R. (2011). A New Framework for Translation Studies and Teaching: A Comprehensive Review of Corpus-Based Translation Studies. Foreign Language Learning Theory and Practice, 1, 8-15.

Yang, H. Z. (2002). An Introduction to Corpus Linguistics. Shanghai: Shanghai Foreign Language Education Press.

Zhen, F. C., \& Zhang, X. (2004). Prospects for the Development of Corpus Linguistics. Foreign Language World, 4, 74-77. 